\title{
Adherence to Current Lipid Guidelines by Physicians in Kuwait
}

\author{
Salwa Alhajji Segun Mojiminiyi \\ Clinical Biochemistry, Mubarak Al-Kabeer Hospital, Jabriya, Kuwait
}

\section{Highlights of the Study}

- This study surveyed and assessed the current standards of care among physicians to better understand barriers to the optimal management of dyslipidemia.

- Three barriers to guideline adherence were reaffirmed in Kuwait: knowledge, attitude, and behavior.

- Recommendations to improve physician adherence include familiarizing physicians with more recent guidelines, improving accessibility, and developing local lipid guidelines.

\section{Keywords}

Clinical sciences · Clinical practice guidelines - Dyslipidemia

\section{Abstract}

Background: Alarmingly high rates of dyslipidemia have been found in the Gulf region in patients presenting with acute coronary syndrome, with the highest being found in Kuwait (37\%). Therefore, it is of utmost importance to treat dyslipidemia promptly and effectively. In an effort to understand the practices of local physicians, the use of evidencebased medicine, and adherence to lipid treatment guidelines, the objective of this study was to survey and assess the current standards of care. Methods: A survey questionnaire, designed to assess physicians' attitudes and practice towards lipid guidelines, was completed by 279 participants and returned between October 2015 and June 2016. Statistical analysis was done using SPSS. Results: Over $90 \%$ of physicians claimed to use lipid guidelines, with the majority rating themselves as knowledgeable. Younger physicians were found to be less knowledgeable and consequently used guidelines less frequently. The most important factor influencing clinical decision-making was the availability of clini-

\begin{tabular}{ll}
\hline karger@karger.com & ( ) 2019 The Author(s) Karger \\
www.karger.com/mpp & Published by S. Karger AG, Basel Open access \\
Karger & $\begin{array}{l}\text { This is an Open Access article licensed under the Creative Commons } \\
\text { Attribution-NonCommercial-4.0 International License (CC BY-NC) } \\
\text { (http://www.karger.com/Services/OpenAccessLicense), applicable to } \\
\text { the online version of the article only. Usage and distribution for com- } \\
\text { mercial purposes requires written permission. }\end{array}$
\end{tabular}

cal guidelines. The majority (72.4\%) of physicians identified time limitation as a key barrier. The most commonly selected lipid guideline in daily practice was a guideline on the treatment of blood cholesterol published by the American College of Cardiology/American Heart Association in 2013. The most common risk assessment tool used was the Framingham risk score. Conclusions: Multiple interventions to improve guideline adherence are proposed in this study. We have taken into account the barriers to adherence, i.e., attitude, behavior, and (most importantly) knowledge, all 3 of which were reaffirmed in our investigation in Kuwait.

$$
\begin{aligned}
& \text { C } 2019 \text { The Author(s) } \\
& \text { Published by S. Karger AG, Basel }
\end{aligned}
$$

\section{Introduction}

Cardiovascular disease (CVD) is the leading cause of mortality worldwide as well as in Kuwait. In Kuwait, 41\% of all mortalities are caused by coronary heart disease, making this a matter of grave importance $[1,2]$. In order to reduce the high rate of cardiovascular mortality, it is essential to alter modifiable traditional risk factors, including obesity, hypertension, hypercholesterolemia, and
Salwa Alhajji

Clinical Biochemistry Laboratory, Mubarak Al-Kabeer Hospital Street 103

Jabriya 43787 (Kuwait)

E-Mail dr.salhajji@gmail.com 
smoking, as recognized by the World Health Organization (WHO). In the Gulf region, alarmingly high rates of dyslipidemia have been found among patients presenting with acute coronary syndrome, with the highest being found in Kuwait (37\%) [3]. It is necessary to emphasize the importance of diagnosing and treating dyslipidemia promptly and effectively in order to achieve optimal risk reduction [4].

Despite plenty of evidence demonstrating ways to reduce atherosclerotic CVD, a gap remains between the standards of care agreed upon and the actual care provided; many candidates eligible for hyperlipidemia treatment do not in fact receive treatment, or else fail to reach optimal lipid levels [5]. For example, previous studies conducted in Kuwait revealed varying degrees of physician compliance with the recommendations of the American College of Cardiology (ACC)/American Heart Association (AHA) regarding blood lipid testing times in patients with myocardial infarction, which, in turn, will ultimately affect the timeliness of treatment [6]. Due to the lack of established national lipid guidelines at the time of this publication, physicians in Kuwait tend to refer to established international guidelines when managing dyslipidemia; these include the 2013 Guideline on the Treatment of Blood Cholesterol by ACC/AHA (otherwise known as ATP IV), Adult Treatment Panel III guidelines (2011), ESC/EAS lipid guidelines (2011), and the NICE guidelines (2014) [7]. In an effort to understand the practices of local physicians' and their use of evidence-based medicine and treatment guidelines, it is important to survey and assess the current standards of care in order to identify obstacles to optimal preventive practices.

Although everyday clinical decisions should predominantly be based on professional excellence and evidence, these are not the sole determinants. Practicing evidencebased medicine involves using the most thorough and upto-date evidence to reach the best clinical decision as opposed to outdated traditional sources [8]. Nevertheless, previous literature has suggested that physicians may base clinical decisions on nonclinical factors irrespective of the evidence. These nonclinical factors may be related to the patient, such as attitudes, preferences, or age. They can also be related to the physician and these include factors such as age, gender, culture, personality, time constraints, and professional interactions in the workplace [8-11]. Although guidelines are not a substitute for clinical judgment, they exist to help guide physicians to a better outcome for the patient on a consistent basis. When a physician treats a patient based on his/her personal expe- rience, he or she may underuse the best available diagnostic and therapeutic tools, with an outcome that is less than optimal.

As studies on the patterns of use of evidence-based medicine in Kuwait are scarce, we evaluated the adherence of physicians to lipid management guidelines in $\mathrm{Ku}$ wait in this study. General thoughts, attitudes, and behaviors regarding medical guidelines were also assessed. We aimed to identify any disparities between the lipid management guidelines that physicians select and the actual course of action taken. Highlighting these shortcomings will identify potential reasons for why patients are not reaching their lipid targets and therefore not benefiting maximally from treatment. Steps can then be taken to achieve greater adherence to guidelines by physicians, which will ultimately reduce costs and improve the quality of treatment [12].

\section{Subjects and Methods}

The study was conducted in Kuwait between October 2015 and June 2016. A total of 279 participants completed the survey questionnaire; the total number of practicing physicians in the governmental sector (all specialties) in 2015 was 8,105 [13, 14]. The main goal of the survey was to evaluate the thoughts and attitudes of physicians regarding the use of clinical guidelines, and the current use of lipid guidelines in Kuwait.

We conducted the survey at multiple locations in the governmental sector. These included general hospitals (Mubarak Al-Kabeer, Jahra, Adan, Farwaniya, Amiri, and Al-Sabah), specialized hospitals (Chest Diseases Hospital), and a subset of primary health care centers from all governorates (Hawalli, Ahmadi, Farwaniya, Capital, and Mubarak Al-Kabeer). The survey was carried out among physicians practicing specialties most likely to encounter dyslipidemia cases; these included Internal Medicine, Endocrinology, Diabetology, Cardiology, Nephrology, Clinical Biochemistry, and Family Medicine. Physicians with varying demographics were encouraged to participate in the study, to eliminate any differences that are encountered when comparing and applying our sample results to the population.

The questionnaire consisted of 3 main sections; the first comprised 6 questions detailing demographic information, the second consisted of 8 questions regarding thoughts and attitudes related to clinical guidelines in general, and the final section contained 6 questions related specifically to lipid guidelines. They were a mixture of closed- and open-ended questions. The answers to the open-ended questions were coded for further analysis.

All respondents were asked how often they use clinical guidelines in daily practice, in addition to more detailed questions about specifically which lipid guidelines they were more likely to adhere or refer to in a clinical environment. The final section also contained some recall questions; these presented a clinical scenario and the participant had to select a course of action which corresponded with their previous answer about which lipid guidelines they adhere to. These questions were designed to evaluate the ac- 
tual knowledge of participants and their familiarity with the subject matter, not just their awareness. A score out of 4 was used to assess how closely their responses matched the lipid guidelines they had stated that they follow. After manual entry of the questionnaire results, we tabulated these according to how often they occurred; for certain key variables, cross-tabulations were made to identify any noteworthy correlations. Statistical analysis was done using the IBM Statistical Package for Social Sciences (2015) and categorical variables were compared using the Pearson $\chi^{2}$ test, with the level of statistical significance set at $p<0.05$.

\section{Results}

A response rate of $79.7 \%$ (279/350 questionnaires were distributed) was achieved between October 2015 and June 2016. Demographic data are presented in Table 1.

\section{Use of Clinical Guidelines}

Most practicing physicians (66.9\%) claimed to consistently use lipid clinical guidelines; only $6.9 \%$ did not use lipid guidelines but were planning to start in the future. Approximately $77.7 \%$ claimed to use lipid guidelines at least $75 \%$ of the time, with the most frequent setting being at diagnosis $(89.5 \%)$, followed by the outpatient/office setting $(85.1 \%)$, and preventative care $(80 \%)$. In the hospital, only $56 \%$ of physicians used lipid guidelines frequently. In cases where guidelines were not used, the most commonly encountered reason (60\%) given was an undetermined diagnosis.

\section{Attitudes towards Guidelines}

Most active physicians regarded themselves as adequately knowledgeable with regard to lipid guidelines, with the majority $(70.5 \%)$ rating themselves as fairly knowledgeable and another $21.8 \%$ as extremely knowledgeable. The most important factor found to influence $82.1 \%$ of physicians' clinical decisions was clinical guidelines. Personal experience (57.8\%) and residency training (55.1\%) also had an important influence on clinical decisions, whilst observing others (20.4\%) and sales representatives (10.9\%) had the least influence. The majority of physicians (96.7\%) agreed that easier access to guidelines would sway them to use these more frequently. Time was found to be another important factor for the routine use of guidelines (72.4\%).

Almost a third of physicians felt that guidelines are too broad or vague to be used as a standard approach (31.8\%) and that their own experience produced better outcomes $(28.5 \%)$. On the other hand, more than half (58.4\%) believed that experimenting with other treatments could
Table 1. Demographics of 279 participants

\begin{tabular}{|c|c|}
\hline Mean age, years (range) & $39.1(25-68)$ \\
\hline \multicolumn{2}{|l|}{ Gender } \\
\hline Male & $124(44.4 \%)$ \\
\hline Female & $153(54.8 \%)$ \\
\hline Not specified & $2(0.7 \%)$ \\
\hline \multicolumn{2}{|l|}{ Nationality } \\
\hline Kuwaiti & $125(44.8 \%)$ \\
\hline Non-Kuwaiti & $154(55.2 \%)$ \\
\hline \multicolumn{2}{|l|}{ Department } \\
\hline Cardiology & $39(14 \%)$ \\
\hline Clinical biochemistry & $8(2.9 \%)$ \\
\hline Diabetology & $18(6.5 \%)$ \\
\hline Endocrinology & $7(2.5 \%)$ \\
\hline Family medicine & $146(52.3 \%)$ \\
\hline Internal medicine & $45(16.1 \%)$ \\
\hline Nephrology & $16(5.7 \%)$ \\
\hline \multicolumn{2}{|l|}{ Workplace } \\
\hline \multicolumn{2}{|l|}{ General hospitals } \\
\hline All & $85(30.4 \%)$ \\
\hline Adan & $11(3.9 \%)$ \\
\hline Amiri & $4(1.4 \%)$ \\
\hline Farwaniya & $7(2.5 \%)$ \\
\hline Jahra & $2(0.7 \%)$ \\
\hline Mubarak Al-Kabeer & $60(21.5 \%)$ \\
\hline Al-Sabah & $1(0.4 \%)$ \\
\hline \multicolumn{2}{|l|}{ Primary health care centers } \\
\hline All & $161(57.7 \%)$ \\
\hline Ahmadi & $13(4.7 \%)$ \\
\hline Capital & $45(16.1 \%)$ \\
\hline Farwaniya & $21(7.5 \%)$ \\
\hline Hawalli & $49(17.6 \%)$ \\
\hline Mubarak Al-Kabeer & $33(11.8 \%)$ \\
\hline \multicolumn{2}{|l|}{ Specialized hospitals } \\
\hline All & $33(11.8 \%)$ \\
\hline Chest Diseases & $31(11.1 \%)$ \\
\hline Kuwait Cancer Control Center & $2(0.7 \%)$ \\
\hline \multicolumn{2}{|l|}{ Length of working experience } \\
\hline$\leq 5$ years & $58(20.8 \%)$ \\
\hline $5-10$ years & $83(29.7 \%)$ \\
\hline $11-20$ years & $70(25.1 \%)$ \\
\hline$\geq 20$ years & $66(23.7 \%)$ \\
\hline Not specified & $2(0.7 \%)$ \\
\hline
\end{tabular}

lead to innovation and treatment advances. More importantly, most physicians believed that having guidelines accessible on their local hospital/clinic data system $(81.8 \%)$ and as a hard copy/paper (61.7\%) would allow them to use the guidelines more regularly, whilst accessing them from a published journal (50\%) or customized e-mail (43.6\%) had less of an impact. 


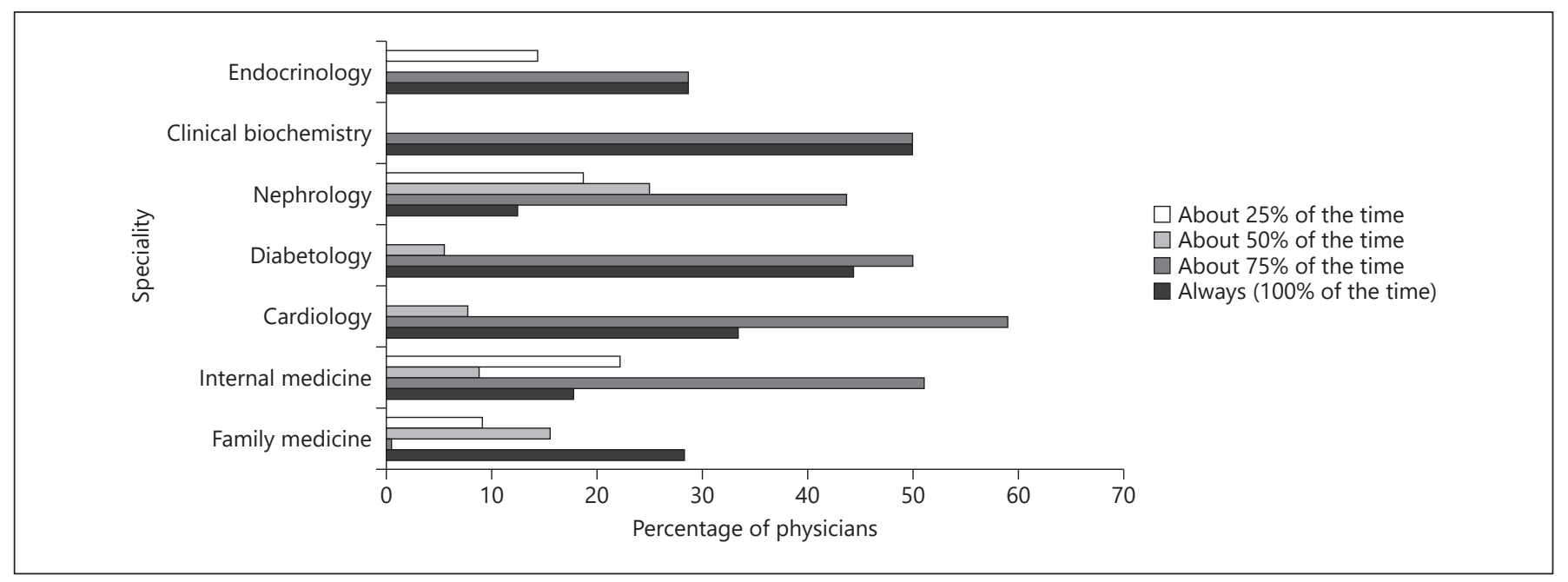

Fig. 1. Frequency of guidelines use by different specialties $(p<0.05)$.

\section{Current Use of Lipid Guidelines for Clinical Decision- Making}

With regards to lipid management, most physicians (83\%) tended to adhere to only 1 set of published guidelines; the other $17 \%$ claimed to follow $>1$ set of guidelines. At the time of analysis, the most commonly selected for clinical decision-making (85\%) was the current 2013 Guideline on the Treatment of Blood Cholesterol by ACC/AHA (ATP IV) and/or the previous version of Adult Treatment Panel III guidelines (2011); 8.2\% followed the ESC/EAS lipid guidelines and 5.4\% used the NICE guidelines. A multitude of reasons contribute to selecting which lipid guideline to follow. Some physicians (30.9\%) base their selection on the applicability of guidelines while others (28.7\%) follow the most recent guidelines published.

Seventy percent of practicing physicians routinely used a single risk assessment tool to classify the cardiovascular risk of their patients, while $7.9 \%$ did not use any risk assessment tool. Of those using a single risk assessment tool, the most commonly used (in order of decreasing frequency) were: the Framingham Coronary Heart Disease Risk Score (60.2\%), ASCVD (10.2\%), SCORE (9.7\%), Q-Risk (9.7\%), WHO (7.1\%), ASSIGN (2.6\%), PROCAM (0.5\%), and ADA (0.5\%). Finally, as previously mentioned, an approximate total score was calculated based on how closely participants' answers to clinical scenarios matched the clinical guidelines that they had previously claimed to follow. The majority of participants (56.6\%) scored poorly $(\leq 25 \%)$ while $38.5 \%$ scored fairly
(50\%), and only $4.9 \%$ had a score of $\geq 75 \%$ in these scenarios.

\section{Differences by Age}

When comparing the frequency of use of guidelines in daily practice, there was a significant difference $(p<$ 0.001 ) between younger and older physicians. A clear trend was noted, i.e., the older the age group, the more frequent the use of guidelines. For example, $90 \%$ of the eldest age group used guidelines most of the time ( $75 \%$ of the time or always), while almost a third (32.3\%) of physicians younger than 30 years of age admitted to using guidelines less often ( $\leq 25 \%$ of the time). In addition, a clear difference $(p<0.001)$ between age groups was observed about the perceived knowledge of guidelines. More than $90 \%$ of those aged $\geq 40$ years rated themselves as fairly knowledgeable. Approximately one-quarter $(25.8 \%)$ of the youngest age group ( $<30$ years) on the other hand, rated themselves as not knowledgeable.

The factors that influenced clinical decisions also differed according to age. The majority (53.3\%) of the younger physicians were strongly influenced by discussion with peers; in contrast, only a quarter $(26.8 \%)$ of older physicians ( $>50$ years) felt that discussion strongly influenced their decisions $(p<0.05)$. However, the influence of sales representatives on clinical decisions was greater as the age of physicians increased. Half of those in the youngest age group and less than a quarter $(22 \%)$ in the eldest age group were not affected by sales representatives $(p<0.05)$. Finally, while the majority of physicians 


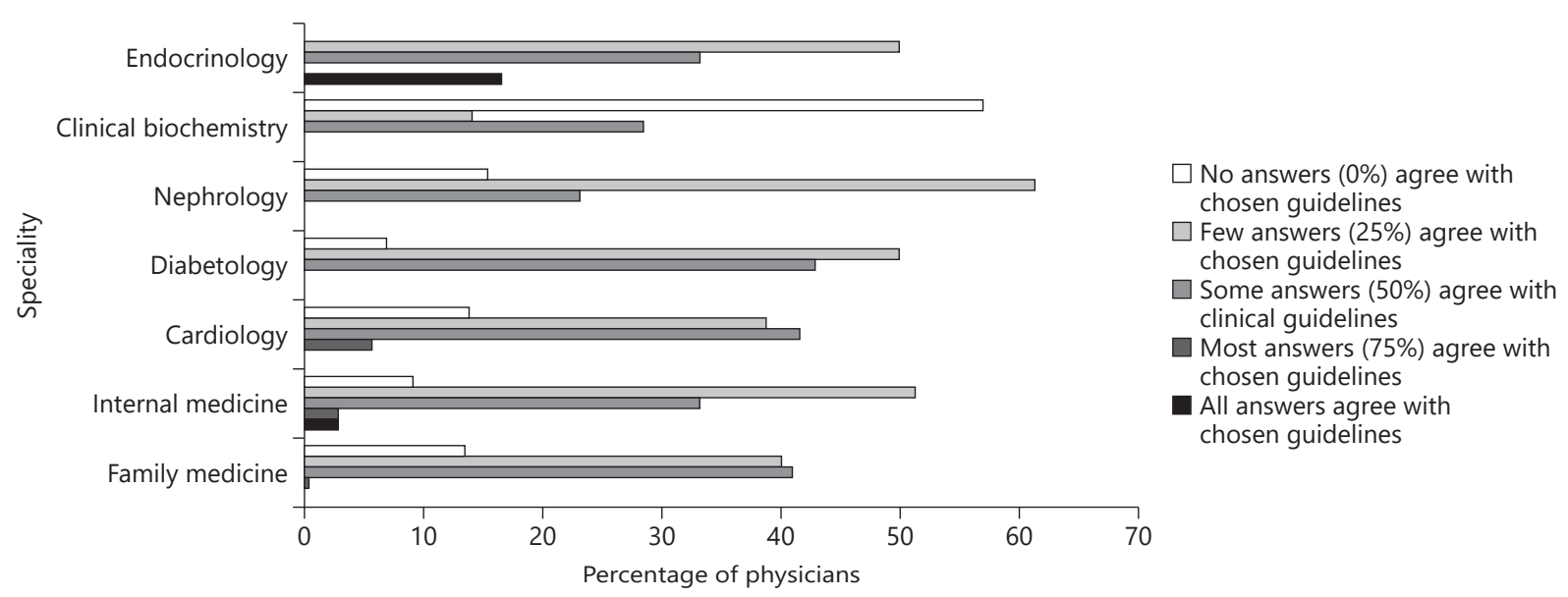

Fig. 2. Total score achieved by different specialties $(p<0.05)$.

Fig. 3. Frequency of guidelines use in different workplaces $(p<0.05)$.

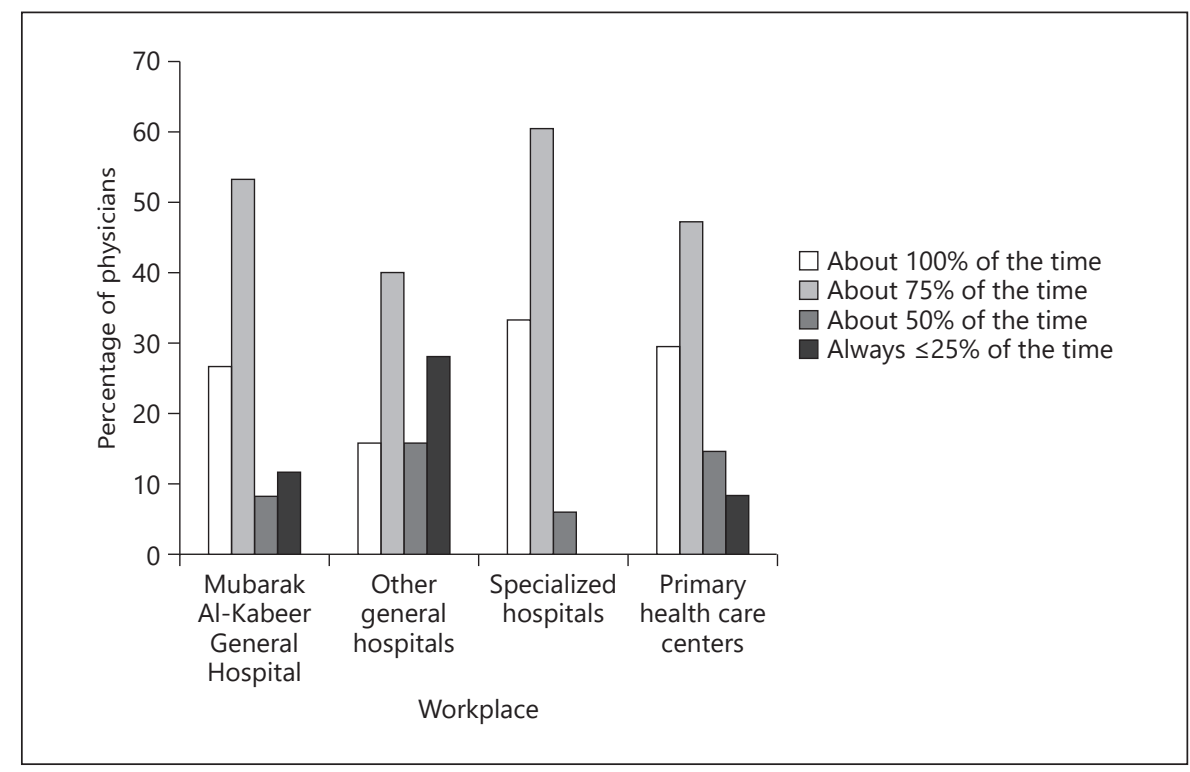

older than 30 years $(\geq 71.4 \%)$ reported selecting 1 risk assessment tool as their primary screening means, a surprisingly high $43.8 \%$ of physicians younger 30 years admitted to not using any risk assessment tool.

\section{Differences by Specialties}

When comparing specialties, all cardiologists, diabetologists, endocrinologists, and clinical biochemists stated that they use lipid guidelines regularly $(p<0.05)$. Figure 1 demonstrates that $31.1 \%$ of internists and $43.8 \%$ of nephrologists adhered to guidelines only $\leq 50 \%$ of the time $(p<0.05)$. Furthermore, the highest percentages of physicians who admitted to not being very knowledgeable with regard to lipid guidelines were also from these very specialties $(p<0.001)$. On the other hand, cardiologists, diabetologists, and clinical biochemists used lipid guidelines at least $50 \%$ of the time. When comparing the total score achieved by different specialties, the responses by endocrinologists to different clinical scenarios were the most compatible with the clinical guidelines they claimed to follow (Fig. 2; $p<0.05$ ). They were followed by cardiologists and family physicians. However, nephrolo- 
gists performed the poorest, with the majority attaining a total score of $<50 \%$.

\section{Differences by Workplace}

There were also some differences observed among physicians working in different centers; $13.3 \%$ of those working in the Mubarak Al-Kabeer General Hospital and $20 \%$ in all other general hospitals admitted to not using any lipid guidelines at all $(p<0.05)$. Physicians working in specialized hospitals or primary health care centers were likely to use lipid guidelines more frequently and were found to use them at least $75 \%$ of the time (Fig. 3; $p<0.05)$. In terms of perceived knowledge about lipid guidelines, all physicians working in specialized hospitals claimed to be fairly or extremely knowledgeable $(p<$ $0.001)$.

\section{Discussion}

Hyperlipidemia is significantly undertreated in $\mathrm{Ku}-$ wait, partly because of multiple nonclinical factors, not previously studied in Kuwait, that play a large role in the practice of physicians. Although the majority of physicians claimed to incorporate clinical lipid guidelines in daily practice and judged themselves as being adequately knowledgeable, this was poorly reflected in their responses to clinical scenarios. There are a number of barriers concerning knowledge, attitudes, and behavior which explain this outcome; these were explored by Scholes [15].

Knowledge barriers may include a lack of familiarity with current guidelines, despite being aware of their existence, in addition to issues with their accessibility [15]. Younger physicians were less likely to use guidelines than older physicians due to less knowledge of the guidelines. Furthermore, despite frequent application of lipid guidelines by cardiologists, diabetologists, endocrinologists, and clinical biochemists, there was an apparent discrepancy in the total familiarity score calculated among these specialties. These findings are comparable to a study conducted in the USA which also found a gap between knowledge and practice, and emphasized the importance of education [16]. Instances such as these may benefit from continuing medical education through lectures, workshops, and conferences, which can lead to better health outcomes [17]. Furthermore, most physicians indicated that improved accessibility through local hospital/clinic data systems or a hard copy/paper would improve their use of guidelines, and consequently their knowledge. It has been proved that the integration of guideline compo-

Adherence to Current Lipid Guidelines by

Physicians in Kuwait nents in the local information system (IT) improves adherence [18]; therefore, incorporating lipid guidelines into the electronic health records can improve health outcomes.

Traditionally, barriers involving attitudes encompass some degree of the disagreement amongst physicians with the approach recommended in certain guidelines, a lack of outcome expectancy, and poor self-efficacy $[15,19]$. Indeed, a significant number of physicians in our study described many guidelines as having low applicability, an attitude barrier, as they were too broad or vague. These findings are comparable with other studies done previously, stressing the importance of developing guidelines which can be individualized for each patient [20]. Furthermore, presenting guideline recommendations in multiple formats, such as algorithms, 1- or 2-page summaries, and electronic Web-based versions may improve user friendliness and serve the varying needs of physicians and patients. Developing local guidelines which are applicable to patients from our demographics is vital, as this will resolve the issue of applicability. Moreover, access to sales representatives has been previously found to encourage adherence to guidelines and, indeed, our study discovered a similar trend, especially in older physicians [21]. Therefore, increasing the access of sales representatives to physicians could improve the adoption of guidelines.

Barriers that are behavioral in nature include constraints on time or resources, patient factors, and guideline factors such as the presence of conflicting guidelines [15]. The study revealed that most physicians felt fewer time constraints would allow them to use lipid guidelines more consistently, as demonstrated in previous studies [22]. In addition, physicians working in specialized hospitals or primary care clinics were more likely to use lipid guidelines than those working in general hospitals; this can be explained by a smaller patient load and fewer time limitations. Health authorities should thus factor this information into the daily workload of physicians and the mean time allocated for consultations, in order to encourage better compliance.

Additional behavioral barriers that play a role in the selection of lipid guidelines include the date of publication, the reputation and experience of the publisher, and preselection by the department. In our study, the majority of physicians chose the 2013 Guideline on the Treatment of Blood Cholesterol by the ACC/AHA, but did not use the risk assessment tool (10-year Atherosclerotic Cardiovascular Disease Risk), also recommended by the ACC/AHA, that is necessary for the assessment of statin intensity requirements. Instead, the majority of physi- 
cians applied the Framingham Coronary Heart Disease Risk Score, which was established much earlier and could be described as more reputable. It is noteworthy that using a combination of lipid guidelines can in fact lead to misinterpretation and ineffective or inadequate treatment as a result of both knowledge and behavior barriers. Once again, these issues can be addressed with reeducation, e.g., academic sessions $[16,17]$.

Secondly, improved accessibility to guidelines should be done by integrating lipid guidelines in the local hospi$\mathrm{tal} /$ clinic electronic health system in addition to different formats of presentation. Encouraging a flow of academic information from sales representatives may also prove beneficial. In addition, the ideal length of consultations to be able to thoroughly evaluate a patient with the aid of guidelines should be taken into account by health officials. Finally, it is imperative that all pertinent specialties collaborate and develop local lipid guidelines to ensure their applicability and the smoother transfer of patients.

Although this study captured the attitudes and behaviors of physicians with regard to lipid guidelines, there were some limitations. It was conducted on a subgroup of physicians, willing to respond to the surveys but from different specialties and workplaces due to a lack of available demographic data; this sample may thus not be an accurate reflection of the entire population of physicians. Nevertheless, we believe our findings have reaffirmed the barriers to adherence that were previously described, and the study may lay the foundation for larger (and quantitative) prospective studies. Our research findings were based on the theoretical responses of physicians to surveys and clinical scenarios, which may not be an accurate representation of real-life behavior. Future research involving true scenarios may depict a more accurate representation of actual physician adherence and knowledge.

\section{Conclusion}

Hyperlipidemia continues to play a large role in cardiovascular mortality and morbidity. The prompt identification and treatment of this disorder is essential to reduce cardiovascular death. Unfortunately, physicians tend to fall short when treating hyperlipidemia. Nonclinical factors were reaffirmed in this study and 3 different barriers were brought forward: knowledge, attitudes, and behavior. Based on these, we have proposed multiple interventions to provide patients with optimal health care. Some recommendations include the reeducation of physicians regarding relevant clinical guidelines and evidence-based medicine, easier access to guidelines including integrating them into electronic medical data records, and, finally, the development of guidelines locally.

\section{Acknowledgement}

The authors thank Dr. Prem Sharma for his support with the statistics, all the physicians who participated in this study, and the supporting staff who helped distribute and collect surveys.

\section{Statement of Ethics}

Ethics approval (297/2015) was obtained from the Ministry of Health in March 2015 and all data were handled with confidentiality.

\section{Disclosure Statement}

The authors have no conflicts of interest to declare.

\section{References}

1 World Health Organization. 2016 Global Health Observatory Data - The Top 10 Causes of Death. Geneva: WHO; 2016.

2 World Health Organization. Noncommunicable Diseases: Country Profiles - Kuwait; 2014. Geneva: WHO; 2016.

3 El-Menyar A, Zubaid M, Shehab A, Bulbanat B, Albustani N, Alenezi F, et al. Prevalence and impact of cardiovascular risk factors among patients presenting with acute coronary syndrome in the Middle East. Clin Cardiol. 2011 Jan;34(1):51-8.
4 Gould AL, Rossouw JE, Santanello NC, Heyse JF, Furberg CD. Cholesterol reduction yields clinical benefit. A new look at old data. Circulation. $1995 \mathrm{Apr} ; 91(8): 2274-82$.

5 Mantel-Teeuwisse AK, Verschuren WM, Klungel OH, Kromhout D, Lindemans AD, Avorn J, et al. Undertreatment of hypercholesterolaemia: a population-based study. $\mathrm{Br} \mathrm{J}$ Clin Pharmacol. 2003 Apr;55(4):389-97.

$6 \mathrm{Al}$ Aqeel A, Mojiminiyi OA, Al Dashti R, Al Ozairi ES. Differences in physician compliance with guideline on lipid profile determination within $24 \mathrm{~h}$ after acute myocardial infarction. Med Princ Pract. 2005 Jan-Feb; 14(1):41-5.
7 Al Sayed N, Al Waili K, Alawadi F, Al-Ghamdi S, Al Mahmeed W, Al-Nouri F, et al. Consensus clinical recommendations for the management of plasma lipid disorders in the Middle East. Int J Cardiol. 2016 Dec;225:26883.

8 Guyatt G, Cook D, Haynes B. Evidence based medicine has come a long way. BMJ. 2004 Oct;329(7473):990-1.

9 Hajjaj FM, Salek MS, Basra MK, Finlay AY. Non-clinical influences on clinical decisionmaking: a major challenge to evidence-based practice. J R Soc Med. 2010 May;103(5):17887. 
10 Schattner A. Are Physicians Decisions Affected by Multiple Nonclinical Factors? Intern Med. 2014.DOI: 10.4172/2165-8048.1000152.

11 Reschovsky JD, Rich EC, Lake TK. Factors Contributing to Variations in Physicians' Use of Evidence at The Point of Care: A Conceptual Model. J Gen Intern Med. 2015 Aug; 30(S3 Suppl 3):S555-61.

12 Kosimbei G, Hanson K, English M. Do clinical guidelines reduce clinician dependent costs? Health Res Policy Syst. 2011 Jun;9(1): 24.

13 World Health Organization. 2015 Global Health Workforce Statistics - The World Bank. Geneva: WHO; 2015.

14 Central Statistical Bureau. Annual Bulletin of the Health Statistics. Kuwait; 2015.

15 Scholes J. Commentary: Cabanna M, et al. Why Don't Physicians Follow Clinical Practice Guidelines? A Framework for Improvement. Nurs Crit Care. 2007;12(4):211-2.
16 Ramsaran E, Preusse P, Sundaresan D, DiMario S, Patel J, Harrison D, et al. Adherence to Blood Cholesterol Treatment Guidelines among Physicians Managing Patients with Atherosclerotic Cardiovascular Disease. Am J Cardiol. 2019 Jul;124(2):169-75.

17 Davis D, O’Brien MA, Freemantle N, Wolf FM, Mazmanian P, Taylor-Vaisey A. Impact of formal continuing medical education: do conferences, workshops, rounds, and other traditional continuing education activities change physician behavior or health care outcomes? JAMA. 1999 Sep;282(9):867-74.

18 Santoro S, Bartman T, Cua C, Lemle S, Skotko B. Use of Electronic Health Record Integration for Down Syndrome Guidelines. Pediatrics. 2018;142(3):e20174119.
19 Cabana MD, Rand CS, Powe NR, Wu AW, Wilson MH, Abboud PA, et al. Why don't physicians follow clinical practice guidelines? A framework for improvement. JAMA. 1999 Oct;282(15):1458-65.

20 Lugtenberg M, Burgers JS, Besters CF, Han D, Westert GP. Perceived barriers to guideline adherence: a survey among general practitioners. BMC Fam Pract. 2011 Sep;12(1):98.

21 Chressanthis GA, Khedkar P, Jain N, Poddar $P$, Seiders MG. Can access limits on sales representatives to physicians affect clinical prescription decisions? A study of recent events with diabetes and lipid drugs. J Clin Hypertens (Greenwich). 2012 Jul;14(7):435-46.

22 Tsiga E, Panagopoulou E, Sevdalis N, Montgomery $\mathrm{A}$, Benos $\mathrm{A}$. The influence of time pressure on adherence to guidelines in primary care: an experimental study. BMJ Open. 2013 Apr;3(4):e002700. 